Aun siendo todas las áreas de interés para profesores, investigadores, planificadores de la Ciencia y Comunidad Científica, en general, el ficherode Ciencias de la Educación por la especificación de los contenidos que recoge, está más ortentado a este colectivo al proceder la literatura analizada de su propio entomo, si bien a la hora de seleccionar la información se puede optar por una materia específica o considerar la globalidad de la producción y poder ver la diversidad de perspectivas, planteamientos, argumentos y contra argumentos que pueden dar lugar a un mayor enriquecimiento de la disciplina.

El análisis de la información de cada documento se estructura en los siguientes campos recuperables:

\section{Autor/es}

Lugar de trabajo: Institución de trabajo del autor/es Núm. de Registro

\section{Título del artículo}

Título de la revista

Datos fuente: Año de la publicación, vol. núm. de págs., ref. bibl. ISSN.

\section{Tipo de publicación}

Lengua en que está escrito el documento original.

Clasificación temática: Sirve para aglutinar la información en la edición de los índices impresos y es también un argumento de búsqueda (cada una de las áreas de la Base Isoc tiene sta propia clasificación).

Localización: Lugar donde se encuentra el documento original.

Descriptores: Conceptualización del contenido del documento, con una media de 5 o 6 descriptores por documento y que sirven además de resumen telegráfico como puntos de acceso para recuperar la información.

Identificadores de personas o instituciones, etc. Topónimos

\section{Legislación}

\section{Periodos históricos}

Resumen: Se incluye en los casos que se acompaña en el documento original o cuando el analista lo considera imprescindible.

La ficha de recogida de datos incluye algún campo más, como es el de palabras candidatas, descriptores secundarios, sentencias y algún otro aspecto que no me parece relevante describirlo aquí.

\section{BASE ICYT}

Producida por el CINDOC, antigno ICYT. Recopila y difunde la información de las siguientes áreas científicas: Agronomía, Astrofísica, Ciencias de la Vida, Ciencias de Ia Tierra, Ciencias del Espacio,
Farmacología, Física, Matemáticas, Química y Tecnología.

\section{BASE IME}

Producida por el Centro de documentación e Informática Biomédica. Recopila y difunde la información de las siguientes áreas científicas: Biomedicina, Administración Sanitaria, Farmacia, Clínica, Medicina Experimental, Microbiología, Psiquiatría, Salud Pública, etc.

\section{BASE CIRBIC}

Analiza y difunde la información de las colecciones existentes en los fondos de las bibliotecas del CSIC y elabora también el catálogo de publicaciones periódicas.

Todas ellas son accesibles también en linea a través de la red IBERPAC o por la red telefónica conmutada, en la sede del Centro de Proceso de Datos del CSIC en la Unidad de Distribución, c/ Pinar, 19. Madrid 28006.

Caferina Anta Centro de Información y Documentación Científica (CINDOC) del CSIC.

\title{
SELECCIONES BIBLIOGRÁFICAS TEMÁTICAS
}

\section{REVISIÓN DE CONCEPCIONES EN EL ÁREA DE LA EVOLUCIÓN}

\section{Grau Sanchez, $R$.}

Cl Diputació, 128, Ir la. Tel. 4548010.

Barcelona 08015

Lugar de trabajo: IES La Llagosta.

\section{Introducción}

La existencia de concepciones espontáneas en la estructura mental de los individuos se extiende a múltiples dominios del conocimiento humano. Estas ideas previas (también preconceptos, representaciones, marcos o concepciones alternativas, y en algunos casos «ciencia intultivas) se han detectado en ciencias experimentales, ciencias sociales $\mathrm{e}$ incluso en psicología (Pozo 1989). Parece indudable su importancia en el proceso de construcción del nuevo conocimiento (Giordan y Martinand 1988).

Son bastante numerosos los trabajos que estudian las concepciones espontáneas de los alumnos en el área de las ciencias experimentales. En todos ellos se constata la existencia de ideas e interpretaciones, con carácter implícito, sobre los fenómenos científicos, que suelen ser contrarias, distintas si más no, a los conceptos y explicaciones científicas que se aceptan en la actualidad.

Existe una cierta documentación sobre las representaciones de los alumnos en el área de la Evolución y en aquellos conceptos de Genética que están imprescindiblemente ligados al aprendizaje de la Evolución (Tabla I). 
Tabla I

Principales estudios sobre Ias representaciones en el área de Ia Evolución y en conceptos de Genética relacionados.

\begin{tabular}{|c|c|c|}
\hline Autor & Año & Contenido \\
\hline Deadman y Kelly & 1978 & Evolución y herencia \\
\hline Brumby & 1979 & Seleccion natural \\
\hline Kargbo et al. & 1980 & $\begin{array}{l}\text { Herencia de los } \\
\text { caracteres }\end{array}$ \\
\hline Longden & 1982 & $\begin{array}{l}\text { Dificultades en } \\
\text { genética }\end{array}$ \\
\hline Hackling y Treagust & 1984 & $\begin{array}{l}\text { Dificuitades en } \\
\text { genética }\end{array}$ \\
\hline Bnimby & 1984 & Selección natural \\
\hline Engel y Wood & 1985a & Adaptación biologica \\
\hline Engel y Wood & $1985 b$ & $\begin{array}{l}\text { Herencia de los } \\
\text { caracteres }\end{array}$ \\
\hline Albaladejo y Lucas & 1988 & Mutación \\
\hline Jiménez y Fernández & 1989 & Selección natural \\
\hline Bishop y Anderson & 1990 & Selección natural \\
\hline
\end{tabular}

\section{Ej estado de ja cuestión}

¿Cuáles son las ideas de los alumnos sobre este tema? Hemos analizado algunos de Ios trabajos más significativos que han sido publicados hasta la fecha (Tabla I) e intentaremos dar una visión global de las concepciones de los alum. nos en esta área. Nos parece conveniente exponer conjuntamente los resultados obtenidos para la yariabilidad y la herencia, por un lado, y los referentes a adaptación y selección, por otro.

\section{Variabilidad y herencia}

* Inicialmente los alumnos (7-13 años) no contemplan la variabilidad intraes. pecifica. Lógicamente no hay para ellos relacion entre variabilidad, selección y adaptación (Deadman y Kelly 1978). En cualquier caso cuando explican la aparición de un nuevo carácter se expresan en términos lamarquistas, haciendo referencia al uso y falta de uso y a la necesidad (aunque sin mostrar un modelo de pensa* miento excesivamente coherente) y acuden a una mezcla de sentido común y empiricismo (aquello que es plausible según su experiencia). Una interpretación mayoritaria contempla la influencia ambiental sobre las caracteristicas he redables (Kargbo et al. 1980).

* Las ideas en esta área evolucionar con la edad y las experiencias. Los alumnos de 13-16 años, aunque identifican una influencia ambiental sobre las características de los organismos (empiricis- mo), ya incluyen explicaciones genéticas. En relación con la herencia de las caracterís- ticas aparecidas bajo influencia ambiental, parece clara para los más pequeños, pero a medida que sehacenmayores se va modificando este pensamiento: las características adquiridas no se heredarían inmediatamente, podrían fijarse genéticamente después de varias generaciones de individuos en los que se repita el cambio fenotípico (Engel y Wood 1985b)

* Hay una cierta controversia en este punto (Hackling y Treagust 1984), pero en cualquier caso, aunque existe una cierta influencia del ambiente en la manifestación de algunas características de los organismos, nunca se heredan las modificaciones adquiridas, y éste no es el razonamiento general de los alumnos a esta edad. Mientras que serían capaces de reconocer laexistencia de la variabilidad intraespecífica, no se entendería el origen por mutación (Engel y Wood 1985b), ni se reconocería su importancia en relación con la evolución, de la transformación gradual de las poblaciones (Bishop y Anderson 1990).

* Parece que a los 14-15 años no está bien establecido el significado científico del concepto de mutación en la mayoría de alumnos, mientras que su uso en términos rigurosos se extendería al $45 \%$ de los alumnos de 17-18 años (Albaiadejo y Lucas 1988). En muchos casos se asociaría su significado a anomalidades, defectos o a otro tipo de cambios biológicos, y pocas veces se relaciona con adaptación o con evolución.

\section{Selección natural y adaptación}

* Para los alumnos más jóvenes, 11-13 años, la selección natural se relaciona con la extinción de algunas especies y la supervivencia de otras. Siempre se centra desde el punto de vista interespecífico. La adaptación sería una respuesta a un cambio ambiental o a problemas de supervivencia. En ambos casos se trata de una necesidad o un intento de perfec. cionarse de los organismos provocado por factores ambientales; existiría una voluntad, deseo o actividad consciente de reaccionar por parte de los organismos frente al medio. Muy pocos alumnos razonan en términos que se acepten actualmente como científicos; su modelo de razonamiento derivaría de una visión antropocéntrica del funcionamiento de Ia naturaleza (Deadman y Kelly 1978).

* Esta situación no varía demasiado entre los alumnos de 13-16 años; para ellos la adaptación se basa en que Ios organis* mos efectuarian conscientemente cambios físicos en respuesta a cambios ambientales, o bien sería una respuesta a una necesidad. S6́lo alrededor del $10 \%$ de Ios alumnos reconoce que la selección natural actúa sobre las poblaciones en el proceso de adaptación, aunque el contexto de la tarea que deben resolver los alumnos, o el tipo de problema que se plantea, puede influir en los resultados (Engel y Wood 1985a). Esta situación se mantiene entre los alumnos mayores, para quienes el mecanismo evolutivo se basa. ría en una mezcia de necesidad, uso y falta de uso y la capacidad de adaptación (Bishop y Anderson 1990). Llama la atención la utílización del concepto de adaptación con significado derivado de contextos cotidianos (un individuo se esfuerza en cambiar), y no en el sentido que en la actualidad le asignan los biblo. gos en el mecanismo evolutivo.

* Estudios con alumnos mayores, 18-20 años, que ya han recibido instrucción formal en estos conceptos, parecen confirmar que el contexto de la cuestión planteada para averiguar sus concepciones puede influiren los resultados (Brumby 1979 y 1984, Jiménez y Femández 1989). En cualquier caso, sólo resuelven correctamente las situaciones entre el $20 \mathrm{y}$ $60 \%$ de los alumnos encuestados. Entre los modelos de razonamiento más extendidos entre los alumnos, destaca el que basa la existencia de cambios evolutivos en la necesidad. Podría decirse que siguen extrapolando desde los cambios vistos a lo largo de la vida de los individuos (que elios llaman adaptativos) para justificar cambios en poblaciones seleccionadas a lo largo de varias generaciones (Brumby 1984). La adaptación continuaría siendo una respuesta a carnbios ambientales, y seguiría existiendo la creencia en que las características ad. quiridas se transmiten a la descendencia; incluso existiría para algunos una inter pretación teleológica implícita, pues los cambios se efectuarían para sobrevivir o con alguna intención. Destaca que un número significativo de alumnos parece utilizar modeios de razonamiento distintos (aceptado y erróneo) en función del contexto, es decír, según el tipo de problema planteado (Jiménez y Fernández 1989).

Para finalizar, habría que añadir algunas consideraciones:

- Bastantes autores destacan las dificul. tades linguísticas derivadas de la precisión del sígnificado de los términos utilizados en Genética (Longden 1982, Engel y Wood 1985b) y de la influencia negativa de los medios de comunicación $y$ otros medios de información como libros de texto e incluso lecturas científ:cas (Jungwirth 1975, Deadman y Kelly 1978, Engel y Wood 1985a, Albaladejo y Lucas 1988, Bishop y Anderson 1990).

- En general se destaca la ausencia de pensamiento probabilístico y del concepto de azar, de importancia central en 
la interpretacion de procesos adaptativos y de la herencia (Deadman y Kelly 1978 , Kargbo et al. 1980, Hackling y Treagust 1984).

- Es difícil para los alumnos la idea del tiempo en la escala evolutiva. Les cuesta comprender que los cambios evolutivos son lentos y transcurren a lo largo de generaciones; al contrario creen que los cambios se suceden en solo una generacion (Brumby 1979).

- Sin Iugar a dudas las concepciones de los alumnos se integran en un modelo de pensamiento más «logico», más fácil de entender, más sencillo (Bishop y Anderson 1990).

\section{Recomendaciones}

Entre las recomendaciones que los autores de las investigaciones hacen a los profesores podemos encontrar algunos puntos en común. Los alumnos desde muy jovenes muestran interés por esta area $y$ en todos Ios niveles muestran ideas bien establecidas sobre el tema, derivadas del aprenoizaje incidental (comics, revistas de divulgacion, televisión, cine, libros de lectura... ) o de su interacción con el aprendizaje formal de los conceptos implicados. Validan constantemente estas ideas o creencias en Ia experiencia diaria. Las concepciones que muestran, en general, no están de acuerdo con ias ideas cientfficas (Deadman y Kelly 1979, Kargbo et al. 1980, Engel y Wood 1985a, Engel y Wood 1985b).

Parece coherente desarrollar materiales para su aprendizaje y así ofrecer oportunidades de estudiar cuestiones por las que muestran interés de una forma más sistemática que los contactos informales con los medios de comuntcación u observaciones ocasionales (Kargbo et al. 1980).

En cualquier caso hay que partir de lo que ya saben los alumnos (Brumby 1984 , Engel y Wood 1985a). Deben explicitar su modelo intuitivo de pensamiento. Para que expresen sus ideas sobre el tema hay que proporcionar suficientes oportunidades estructuradas. Hay que utilizarlas como punto de partida de discusiones en pequeños grupos. Centrarse en problemas que sean familiares a la mayoría de alumnos e intentar buscar caminos que incorporen las ideas derivadas de la experiencia diaria a las lecciones de Biología (Engel y Wood 1985b). Las estrategias didácticas deben tener como componente esencial la discusión de problemas, mejor si conectan Ciencia y Sociedad (problemas derivados de contextos cotifianos) y proporcionan situaciones de aplicación en casos relacionados con los alumnos (Brumby 1984).

La historia de la Biología nos puede ayudar de dos formas. Primero en la determinación de cuáles pueden ser los principales problemas en el significado de los conceptos a partir del análisis de las mayores dificultades que tuvo que resolver la ciencia (Brumby 1984). En segundo lugar debe considerarse la posibilidad de utilizar el análisis de la historia del conocimiento científico en este campocomouna estrategia didáctica(Engel y Wood 1985a).

\section{Referencias bibliográficas}

Albaladejo, C.y Lucas, A.M., 1989. Pupils' meaning for «mutation», Journal of Biological Education, Vol. 22 (3), pp. 215-219.

Bishop, B. y Anderson, C.W., 1990. Student conceptions of natural selection and its role in evolution, Journal of Research in Science Teaching, Vol. 27 (5), pp. 415-427.

Brumby, M., 1979. Problem in learning the concept of natural selection, Journal of Biological Education. Vol. 13 (2), pp. 119-122.

Brumby, M., 1984. Misconceptions about the concept of Natural Selection by medical biology students, Science Education, Voi. 68 (4), pp. 493-503.
Deadman, J.A. y Kelly, P.J., 1978. What do secondary school boys understand about evolution and heredity before they are taught the topics?, Journal of Biological Education, Vol. I2(1), pp. 7-15.

Engel, E. y Wood, C., 1985a. How secondary students interpret instances of biological adaptation, Journal of Biological Education, Vol. 19 (2), pp. $125-130$.

Engel, E. y Wood, C., 1985b. Children's understanding of inheritance, Journal of Biological Education, Vol. 19 (4), pp. 304-310.

Giordan, A. y Martinand, J.L., 1988. Etat des recherches sur les conceptions des apprenants a propos de la Biologie. Annales de didactique des Sciences, Vol. 140 , pp. II -63 .

Hackling, M. y Treagust, D., 1984. Research data necessary for meaningful review of grade ten high school genetics curricula, Journal of Research in Science Teaching, Vol. 21 (2), pp. 197-209.

Jiménez, M.P. y Fernández, J., 1989. ¿Han sido seleccionados o se han acostumbrado?, Infancia y aprendizaje, Vol.47, pp. 67-81.

Jungwirth, E., 1975. Preconceived adaptation and inverted evolution, The Australian Science Teachers Journal, Vol. 21 (2), pp. 95-100.

Kargbo, D., Hobbs, E.D. y Erikson, G., 1980. Children's beliefs about inherited characteristics, Journal of Biological Education, Vol. 14(2), pp. 137-146.

Longden, B., 1982. Genetics, are there inherent learning dificulties?, Journal of Biological Education, Vol. 16 (2), pp. $135-140$.

Pozo, J.I., 1989. Teorías cognitivas del aprendizaje. (Morata: Madrid). 